UCLA $/ 98 /$ TEP $/ 30$

\title{
Pulsar kicks from neutrino oscillations
}

\author{
Alexander Kusenko \\ Department of Physics and Astronomy, UCLA, Los Angeles, CA 90095-1547 \\ Gino Segrè \\ Department of Physics, University of Pennsylvania, Philadelphia, PA 19104
}

(November, 1998)

\begin{abstract}
Neutrino oscillations can explain the observed motion of pulsars. We show that two different models of neutrino emission from a cooling neutron star are in good quantitative agreement and predict the same order of magnitude for the pulsar kick velocity, consistent with the data.

PACS numbers: 97.60.Gb, 14.60.Pq
\end{abstract}

\section{INTRODUCTION}

We recently suggested [1,2] that the observed proper motions of pulsars [3] may be the result of neutrino oscillations in the hot neutron star born in a supernova explosion. Neutrinos are the only significant cooling agents during the first 10 seconds after the onset of the supernova, and they carry away most of the energy liberated in the gravitational collapse, $\sim 10^{53} \mathrm{erg}$. A $1 \%$ asymmetry in the distribution of neutrinos can account for the measured pulsar velocities $\sim 500 \mathrm{~km} / \mathrm{s}$.

Neutrino oscillations in medium are affected by the polarization effects due to magnetic field 肺. Consequently, the strong magnetic field of a neutron star can affect the depth at which the neutrino conversions take place. The points of the Mikheev-Smirnov-Wolfenstein resonance lie on a surface that is not concentric with the star. For a certain range of neutrino masses, this creates an asymmetry in the distribution of momenta of the outgoing neutrinos. The $\nu_{\mu, \tau} \leftrightarrow \nu_{e}$ oscillations [1], as well as the sterile-to-active neutrino conversions [2], can give the pulsar a kick consistent with the observation provided that the magnetic field inside the star is of order $10^{14}-10^{15} \mathrm{G}$. Other types of neutrino conversions could also produce a similar effect [0].

Our explanation [1.2] uses the fact that different neutrino species have different opacities in nuclear matter. The charged-current interactions are responsible for the difference in the mean free paths of $\nu_{e}$ and $\nu_{\mu, \tau}$. They are also the cause of the matter-enhanced $\nu_{e} \leftrightarrow \nu_{\mu, \tau}$ conversions. In the case of sterile neutrinos, both charged and neutral currents contribute to the difference in the opacities. The analyses of Refs. [1.2, 2. 5 was based on a simplified model which, as we will see, gave the right order-of-magnitude estimate for the pulsar velocity.

Recently, a different model for the neutrino emission was used to calculate the kick [6] due to the active neu- trino oscillations]. The result obtained in Ref. [6] was incorrect because the neutrino absorption $\nu_{e} n \rightarrow e^{-} p^{+}$was neglected and also because the different neutrino opacities were assumed to be equal to each other. We emphasize that in the absence of charged-current interactions the kick from the active neutrino oscillations [1] should vanish.

We will show that, after the charged-current interactions are included, the two models are, in fact, in good agreement, as they should be.

\section{HARD NEUTRINOSPHERES}

Our calculations [1,2] employed a model with a sharp neutrinosphere, such that the neutrinos of a given type were assumed trapped inside and free-streaming outside. Several subsequent analyses [5] used the same model to calculate the kick to the pulsar from the neutrinos whose opacities changed on their passage through matter due to oscillations.

In this model one assumes that the luminosity of the emitted neutrinos obeys the Stefan-Boltzmann law and is proportional to $T^{4}$.

This model is admittedly simplistic. However, it appears to work well insofar as predicting the order of magnitude of the kick.

\section{SOFT NEUTRINOSPHERES}

Let us now consider the Eddington model for the atmosphere which was used by Schinder and Shapiro [7 to

\footnotetext{
${ }^{1}$ The active-to-sterile neutrino conversions of Ref. [2] are assumed to take place at much higher densities than those discussed in Ref. 6].
} 
describe the emission of a single neutrino species. We will generalize it to include several types of neutrinos.

In the diffusion approximation, the distribution functions $f$ are taken in the form [7]:

$$
f_{\nu_{i}} \approx f_{\bar{\nu}_{i}} \approx f^{e q}+\frac{\xi}{\Lambda_{i}} \frac{\partial f^{e q}}{\partial m}
$$

where $f^{e q}$ is the distribution function in equilibrium, $\Lambda_{i}$ denote the respective opacities, $m$ is the column mass density, $m=\int \rho d x, \xi=\cos \alpha$, and $\alpha$ is the normal angle of the neutrino velocity to the surface. At the surface, one imposes the same boundary condition for all the distribution functions, namely

$$
\begin{aligned}
& f_{\nu_{i}}(m, \xi)=0, \quad \text { for } \xi<0, \\
& f_{\nu_{i}}(m, \xi)=2 f^{e q}, \text { for } \xi>0 .
\end{aligned}
$$

However, the differences in $\Lambda_{i}$ produce unequal distributions for different neutrino types.

Generalizing the discussion of Refs. [6, [7] to include six flavors, three neutrinos and three antineutrinos, one can write the energy flux as

$$
F=2 \pi \int_{0}^{\infty} E^{3} d E \int_{-1}^{1} \xi d \xi \sum_{i=1}^{3}\left(f_{\nu_{i}}+f_{\bar{\nu}_{i}}\right)
$$

We will assume that $\Lambda_{i}=\Lambda_{i}^{(0)}\left(E^{2} / E_{0}^{2}\right)$.

We use the expressions for $f_{\nu_{i}}$ from equation (11). Changing the order of differentiation with respect to $m$ and integration over $E$ and $\xi$, and using the fact that $f^{e q}$ is isotropic, we arrive at the result similar to that of Ref. [7]:

$$
F=\frac{2 \pi^{3}}{9} E_{0}^{2}\left[\sum_{i=1}^{3} \frac{2}{\Lambda_{i}^{(0)}}\right] \frac{\partial T^{2}}{\partial m} .
$$

The basic assumption of the model is that flux $F$ is conserved. In other words, the neutrino absorptions $\nu_{e} n \rightarrow e^{-} p^{+}$are neglected. Since the sum in brackets, as well as the flux $F$ are treated [7] as constants with respect to $m$, one can solve for $T^{2}$ :

$T^{2}(m)=\frac{9}{2 \pi^{3}} E_{0}^{-2}\left[\sum_{i=1}^{3} \frac{2}{\Lambda_{i}^{(0)}}\right]^{-1} F m+\left(\frac{30}{7 \pi^{5}} F\right)^{1 / 2}$

Swapping the two flavors in equation (5) leaves the temperature unchanged in the Eddington approximation. Hence, neutrino oscillations do not alter the temperature profile in this approximation?.

\footnotetext{
${ }^{2}$ It was also assumed in our earlier calculations [1.2] that neutrino oscillations do not have a significant effect on the temperature profile. We then took the spectral temperature of free-streaming neutrinos to be equal to matter temperature at their respective neutrinospheres.
}

We will now include the absorptions of neutrinos.

Some of the electron neutrinos are absorbed on their passage through the atmosphere thanks to the chargedcurrent process

$$
\nu_{e} n \rightarrow e^{-} p^{+}
$$

The cross section for this reaction is $\sigma=1.8 G_{F}^{2} E_{\nu}^{2}$, where $E_{\nu}$ is the neutrino energy. The total momentum transfered to the neutron star by the passing neutrinos depends on the energy.

Both numerical and analytical calculations show that the muon and tau neutrinos leaving the core have much higher mean energies than the electron neutrinos [8.97. Below the point of MSW resonance the electron neutrinos have the mean energies $\approx 10 \mathrm{MeV}$, while the muon and tau neutrinos have energies $\approx 25 \mathrm{MeV}$.

The origin of the kick in this description is that the neutrinos spend more time as energetic electron neutrinos on one side of the star than on the other side, hence creating the asymmetry. Although the temperature profile remains unchanged in Eddington approximation, the unequal numbers of neutrino absorptions push the star, so that the total momentum is conserved.

Below the resonance $E_{\nu_{e}}<E_{\nu_{\tau, \mu}}$. Above the resonance, this relation is inverted. The energy deposition into the nuclear matter depends on the distance the electron neutrino has traveled with a higher energy. This distance is affected by the direction of the magnetic field relative to the neutrino momentum.

We assume that the resonant conversion $\nu_{e} \leftrightarrow \nu_{\tau}$ takes place at the point $r=r_{0}+\delta(\phi) ; \delta(\phi)=\delta_{0} \cos \phi$. The position of the resonance depends on the magnetic field $B$ inside the star [1]:

$$
\delta_{0}=\frac{e \mu_{e} B}{2 \pi^{2}} / \frac{d N_{e}}{d r},
$$

where $N_{e}=Y_{e} N_{n}$ is the electron density and $\mu_{e}$ is the electron chemical potential.

Below the resonance the $\tau$ neutrinos are more energetic than the electron neutrinos. The oscillations exchange the neutrino flavors, so that above the resonance the electron neutrinos are more energetic than the $\tau$ neutrinos. The number of neutrino absorptions in the layer of thickness $2 \delta(\phi)$ around $r_{0}$ depends on the angle $\phi$ between the neutrino momentum and the direction of the magnetic field. Each occurrence of the neutrino absorption transfers the momentum $E_{\nu_{e}}$ to the star. The difference in the numbers of collisions per electron neutrino between the directions $\phi$ and $-\phi$ is

$$
\begin{aligned}
\Delta k_{e} / E_{\nu_{e}} & =2 \delta(\phi) N_{n}\left[\sigma\left(E_{1}\right)-\sigma\left(E_{2}\right)\right] \\
& =1.8 G_{F}^{2}\left[E_{1}^{2}-E_{2}^{2}\right] \frac{\mu_{e}}{Y_{e}} \frac{e B}{\pi^{2}} h_{N_{e}} \cos \phi,
\end{aligned}
$$

where $h_{N_{e}}=\left[d\left(\ln N_{e}\right) / d r\right]^{-1}$.

We use $Y_{e} \approx 0.1, E_{1} \approx 25 \mathrm{MeV}, E_{2} \approx 10 \mathrm{MeV}$, $\mu_{e} \approx 50 \mathrm{MeV}$, and $h_{N_{e}} \approx 6 \mathrm{~km}$. After integrating over 
angles and taking into account that only one neutrino species undergoes the conversion, we obtain the final result for the asymmetry in the momentum deposited by the neutrinos:

$$
\frac{\Delta k}{k}=0.01 \frac{B}{2 \times 10^{14} \mathrm{G}}
$$

which agrees with the earlier estimates $]^{3}$ [1, 10.

Neutrinos also lose energy by scattering off the electrons. Since the electrons are degenerate, the final-state electron must have energy greater than $\mu_{e}$. Therefore, electron neutrinos lose from 0.2 to 0.5 of their energy per collision in the neutrino-electron scattering. However, since $N_{e} \ll N_{n}$, this process can be neglected.

One may worry whether the asymmetric absorption can produce some back-reaction and change the temperature distribution inside the star altering our result (10). If such effect exists, it is beyond the scope of Eddington approximation, as is clear from equation (5). The only effect of the asymmetric absorption is to make the star itself move, in accordance with the momentum conservation. This is the origin of the kick (10).

Of course, in reality the back-reaction is not exactly zero. The most serious drawback of Eddington model, pointed out in Ref. [7], is that diffusion approximation breaks down in the region of interest, where the neutrinos are weakly interacting. Another problem has to do with inclusion of neutrino absorptions and neutrino oscillations [7]. However, to the extent we believe this approximation, the pulsar kick is given by equation (10).

\section{COMPARISON OF THE TWO MODELS}

We have juxtaposed two models for neutrino transfer. One of them assumes that neutrinos are trapped inside a sharply defined neutrinosphere, but that they are free-streaming outside. In this model, the absorption of neutrinos outside the neutrinosphere is neglected and the asymmetry arises from the change in the position of the neutrinosphere due to neutrino oscillations.

The other model considers the propagation of the neutrinos through the atmosphere interpolating between the region where the neutrinos are trapped and that in which they are free-streaming. Here the neutron star receives a kick from the unequal numbers of reactions $\nu_{e} n \rightarrow p^{+} e^{-}$ on either side. The higher-energy neutrinos spend more time as electron neutrinos on one side of the star than on the other side. Therefore, the passage of neutrinos creates an uneven drag to the star.

\footnotetext{
${ }^{3}$ We note in passing that we estimated the kick in Refs. [1, 2$]$ assuming $\mu_{e} \approx$ const. A different approximation, $Y_{e} \approx$ const, gives a somewhat higher prediction for the magnitude of the magnetic field [10].
}

Incidentally, the Stefan-Boltzmann relation between the luminosity and temperature is, of course, present in both models. In the diffusion approximation, equation (5) implies that $F \propto T^{4}$ at $m=0$.

In both models the kick is a manifestation of the unequal neutrino opacities which are caused by the chargedcurrent interactions.

The two models are in good quantitative agreement.

\section{CONCLUSION}

The neutrino oscillations can be the explanation of the pulsar motions. Although many alternatives have been examined, all of them fail to explain the large magnitudes of the pulsar velocities. Parity violation effects recently claimed to explain the kicks [1] turned out to be irrelevant because they wash out in statistical equilibrium and produce no appreciable asymmetry in the neutrino luminosity [12]. Other proposed mechanisms required unusually high neutrino magnetic moments [13] or some exotic interactions. The oscillations appear to be the only viable explanation at present.

If the pulsar kick velocities are due to $\nu_{e} \leftrightarrow \nu_{\mu, \tau}$ conversions, one of the neutrinos must have mass $\sim 100 \mathrm{eV}$ (assuming small mixing) and must decay on the cosmological time scales not to overclose the Universe [1]. This has profound implications for particle physics hinting at the existence of Majorons [14 or other physics beyond the Standard Model that can facilitate the neutrino decay.

If the active-to-sterile neutrino oscillations [2] are responsible for pulsar velocities, the prediction for the sterile neutrino to have a mass of several $\mathrm{keV}$ is not in contradiction with any of the present bounds. In fact, the $\sim \mathrm{keV}$ mass sterile neutrino has been proposed as a dark-matter candidate 15 .

To summarize, two different models of neutrino emission predict the same order of magnitude for the pulsar birth velocity caused by neutrino oscillations in the cooling neutron star.

[1] A. Kusenko and G. Segrè, Phys. Rev. Lett. 77, 4872 (1996).

[2] A. Kusenko and G. Segrè, Phys. Lett. B 396, 197 (1997).

[3] See, e.g., A. G. Lyne and D. R. Lorimer, Nature 369 (1994) 127; J. M. Cordes and D. F. Chernoff, Astrophys. J. 505, 315 (1998); B. M. S. Hansen and E. S. Phinney, Mon. Not. R. Astron. Soc. 291, 569 (1997); C. Fryer, A. Burrows, and W. Benz, Astrophys. J. 496, 333.

[4] V. B. Semikoz and J. W. F. Valle, Nucl. Phys. B 425, 651 (1994); ibid. 485, 545 (1997); J. F. Nieves and P. B. Pal, Phys. Rev. D40 1693 (1989); J. C. D’Olivo, J. F. Nieves 
and P. B. Pal, ibid., 3679 (1989); J. C. D'Olivo, J. F. Nieves and P. B. Pal, Phys. Rev. Lett., 64, 1088 (1990); H. Nunokawa, V. B. Semikoz, A. Yu. Smirnov, and J. W. F. Valle Nucl. Phys. B 501, 17 (1997).

[5] E.Kh. Akhmedov, A. Lanza and D.W. Sciama, Phys. Rev. D 56, 6117 (1997); C. W. Kim, J. D. Kim, and J. Song, Phys. Lett. B 419 (1998) 279; D. Grasso, FTUV-98-13, astro-ph/9802060; D. Grasso, H. Nunokawa, and J.W.F. Valle, Phys. Rev. Lett. 81, 2412 (1998); R. Horvat, hep-ph/9806380.

[6] H.-Th. Janka and G. G. Raffelt, astro-ph/9808099.

[7] P.J. Schinder and S.L. Shapiro, Astrophys. J. 259, 311 (1982).

[8] H. Suzuki, in: Physics and Astrophysics of Neutrinos, ed. by M. Fukugita and A. Suzuki (Springer-Verlag, Tokyo, 1994).

[9] R.W. Mayle, J.R. Wilson and D.N. Schramm, Astrophys. J. 318, 288 (1987); S.W. Bruenn, Phys. Rev. Lett. 59, 938 (1987).

[10] Y.-Z. Qian, Phys. Rev. Lett. 79, 2750 (1997); A. Kusenko and G. Segrè, Phys. Rev. Lett. 792751 (1997).

[11] C.J. Horowitz and G. Li, Phys. Rev. Lett. 80, 3694 (1998); D. Lai and Y.-Z. Qian, astro-ph/9802344, Astrophys. J. 495, L103 (1998); ibid. 501, L155 (1998); H.-Th. Janka, in Proceedings of "Neutrino Astrophysics", ed. M. Altmann et al., Tech. Univ. München, Garching, 1998 atro-ph/9801320.

[12] A. Kusenko, G. Segrè, and A. Vilenkin, Phys. Lett. B 437, 359 (1998); P. Arras and D. Lai, astro-ph/9806285.

[13] M. B. Voloshin, Phys. Lett. B 209 (1988) 360.

[14] For a recent review, see, e. g., R. D. Peccei, hep$\mathrm{ph} / 9807516$.

[15] S. Dodelson and L. M. Widrow, Phys. Rev. Lett. 72, 17 (1994); G. M. Fuller and X. Shi, astro-ph/9810076. 